Techniques \& Culture

Revue semestrielle d'anthropologie des techniques

$59 \mid 2012$

Itinéraires de coquillages

\title{
Des coquillages de valeur
}

Shells of value

\section{Francis Dupuy}

\section{(2) OpenEdition}

Journals

Édition électronique

URL : https://journals.openedition.org/tc/6568

DOI : $10.4000 /$ tc. 6568

ISSN : 1952-420X

Éditeur

Éditions de l'EHESS

Édition imprimée

Date de publication : 15 décembre 2012

Pagination : 96-109

ISBN : 978-2-7351-1534-1

ISSN : 0248-6016

\section{Référence électronique}

Francis Dupuy, « Des coquillages de valeur», Techniques \& Culture [En ligne], 59 | 2012, mis en ligne le 15 décembre 2015, consulté le 29 septembre 2022. URL : http://journals.openedition.org/tc/6568 ;

DOI : https://doi.org/10.4000/tc.6568 


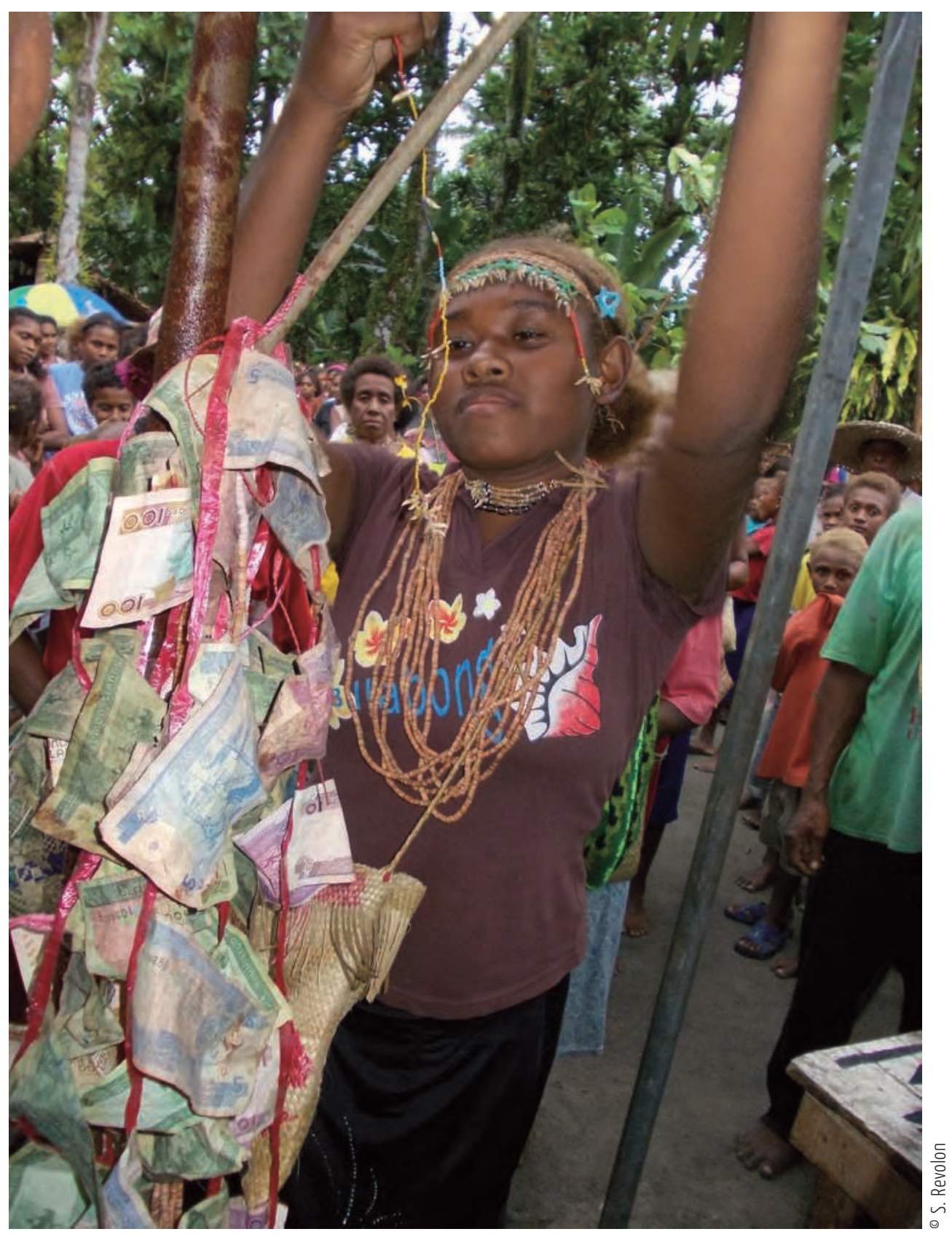


Francis Dupuy

Université de Poitiers/EREA-LESC

francis.dupuy@univ-poitiers.fr
Itinéraires de coquillages - 1

Techniques \& Culture 59, 2012/2: 96-109

\section{DES COQUILLAGES DE VALEUR}

Parmi tous les objets et supports matériels divers avec lesquels les hommes en société ont su fabriquer des monnaies - ce qu'il est convenu d'appeler des monnaies « primitives » ou « traditionnelles »-, les coquillages occupent sans nul doute une place de choix (Einzig 1949, Quiggin 1949, Dupuy 2008). En diverses parties du monde - l'Océanie au premier chef, mais aussi l'Afrique d'une autre manière ${ }^{1}$ - des coquillages les plus divers et sous les formes les plus variées ont servi d'équivalents à d'autres biens matériels lors de relations d'échange mais aussi de substituts à des humains dans le cadre d'échanges matrimoniaux ou de compensations mortuaires. C'est d'ailleurs cette fascinante plurifonctionnalité qui rend si difficile la compréhension de ces « phénomènes monétaires » ${ }^{2}$.

Nous examinerons ici, à travers quelques exemples significatifs, d'abord les procédures sociales, culturelles et économiques permettant de transformer de simples coquillages en objets de valeur et/ou en monnaie, puis la réussite fulgurante de l'un des coquillages les plus répandus au monde, le cauri, et enfin les aventures de ce dernier parvenu en Amérique du Sud.

\section{La valeur des coquillages}

Il ne suffit pas d'un coquillage pour « faire » une monnaie. Une société - quelle qu'elle soit - qui entend faire d'un coquillage - quel qu'il soit - une monnaie fait subir à ce 
Brasses de perles de coquillage rouges et blanches. Les premières sont les plus précieuses, équivalent à 1000 SDB (dollar Salomon) soit environ 100 euros. Leur longueur est la même que la distance séparant les deux mains lorsque les bras sont écartés perpendiculairement au corps. Aorigi, Est des îles Salomon, juillet 2011. dernier une métamorphose. Cette métamorphose opère sur deux leviers simultanés et complémentaires. En premier lieu, elle réalise une valeur socialement et culturellement construite; ce traitement passe le plus souvent, comme nous allons le voir, par une rareté, réelle ou artificiellement fabriquée. En second lieu, elle crée un code social sur lequel les acteurs sociaux s'entendent pour reconnaître à ce coquillage une certaine valeur; c'est l'aspect que l'on pourrait dire fiduciaire, inhérent à toute monnaie ${ }^{3}$. Nous ne confondons pas ici valeur et richesse, même si les deux, évidemment, sont intimement liées. Dans nos sociétés industrielles (i. e. dans le mode de production capitaliste), c'est le travail, et plus encore le surtravail, générateur de plus-value, qui produit la richesse. Dans les sociétés dont il est question ici, la logique en œuvre est de fabriquer de la valeur cristallisée dans des objets initialement communs pour en faire des objets précieux: les items supports de richesse ne sont pas des biens matériels ordinaires, mais des biens que l'on rend extra-ordinaires d'abord en les coupant de leur source de provenance puis en «fantasmant » leur origine. La valeur ainsi créée, nous la qualifierons, faute de meilleur terme, de symbolique. Le voyage, dès lors, sera constitutif de la valeur des coquillages faisant office de monnaies.

Ainsi donc, l'une des astuces les plus usitées afin de donner de la valeur à un objet, quel qu'il soit, est de le rendre rare d'une façon ou d'une autre. Et l'une des façons assez courantes de créer cette rareté est de prendre un objet « venu d'ailleurs ». Beaucoup de monnaies de coquillages proviennent effectivement d'ailleurs (Dupuy 2009). C'est le cas pour la plupart de celles que l'on rencontre dans les archipels orientaux de la NouvelleGuinée (Nouvelle-Bretagne, Nouvelle-Irlande, îles Salomon...). Nous prendrons quelques exemples bien connus dans la littérature anthropologique.

Un premier cas intéressant nous est offert par Langalanga, une petite communauté mélanésienne vivant sur quelques îlots situés au large de la côte occidentale de Malaita,

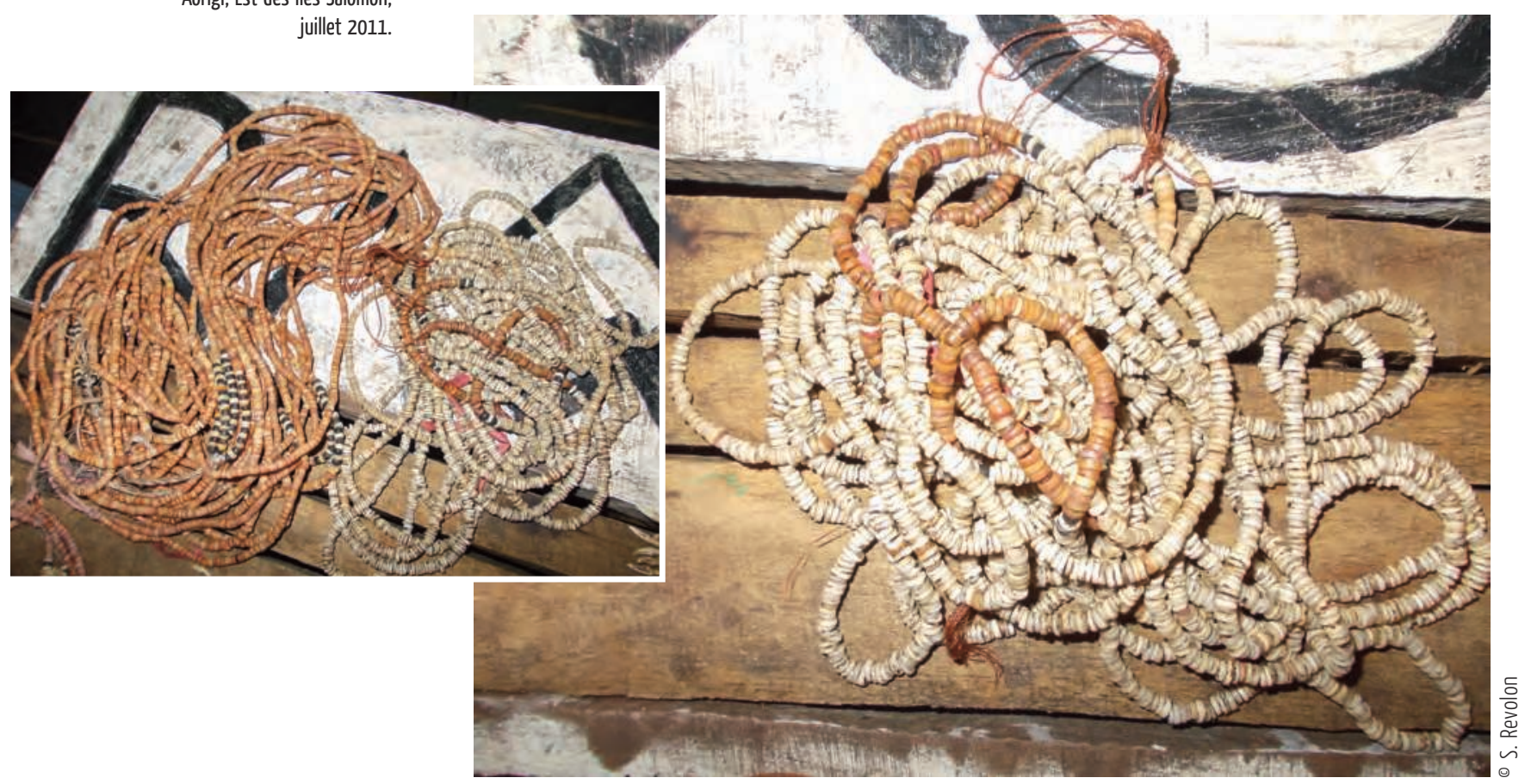


laquelle s'est fait une spécialité de la fabrication d'une monnaie composée de petits disques de divers coquillages pêchés dans le lagon environnant qui sont réunis en brasses (Cooper 1971, Connell 1977, Guo 2007). La ressource est étroitement contrôlée; elle relève de droits coutumiers portant sur le lagon. Le travail de fabrication incombe tout particulièrement aux femmes. Ces monnaies sont ensuite acheminées hors des lieux de fabrication pour se diffuser dans toute une vaste région (Malaita, Guadalcanal, Rossel, Santa Isabel, Choiseul, Bougainville et jusqu'en Nouvelle-Irlande...). La première étape de l'échange intervient entre les habitants de Langalanga et quelques communautés voisines attitrées afin de se procurer des biens de consommation manquants, en particulier

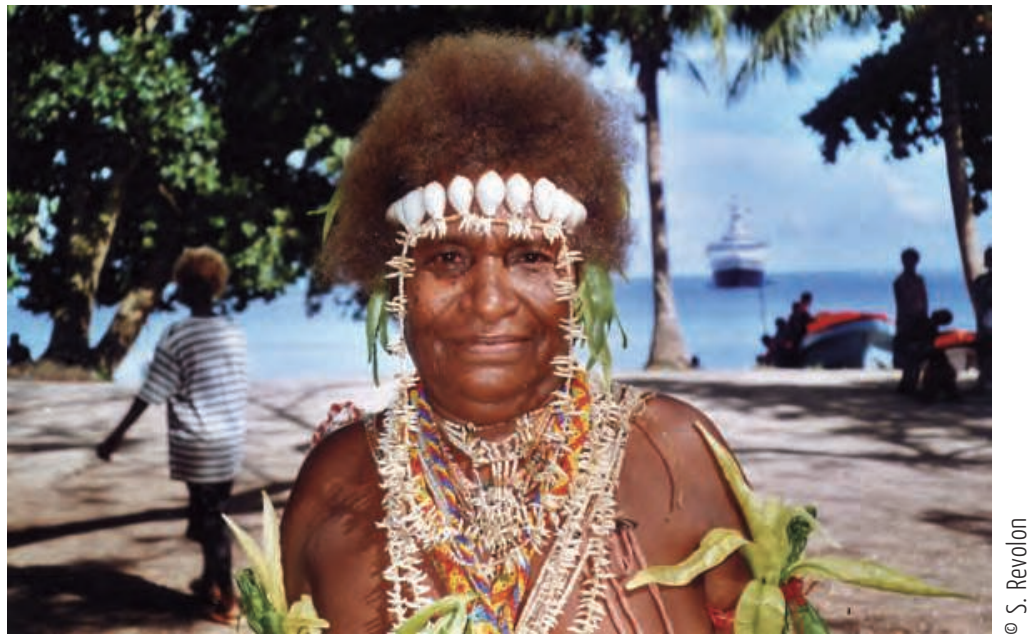
des produits horticoles et des porcs nécessaires au déroulement de fêtes et de rituels. Lorsqu'elles quittent Langalanga, ces brasses de coquillages ont déjà, indéniablement, une certaine valeur de monnaie. Mais ce qui construit la force symbolique et la pleine valeur « monétaire » de ces brasses de coquillages, c'est leur long périple à travers des réseaux d'échange complexes et à multiples ramifications. Ainsi, lorsque ces brasses parviennent à Bougainville ou en Nouvelle-Irlande, leur origine s'est perdue au loin et plus ou moins mythifiée - de sorte que celles-ci paraissent venues d'un « ailleurs » inconnu et insaisissable pour les gens qui les reçoivent.

Un autre exemple est celui des pearlshells (gros coquillages nacrés) en NouvelleGuinée, qui proviennent des régions littorales et qui gravitent de partenaire en partenaire et de circuit d'échange en circuit d'échange, jusqu'à parvenir dans l'intérieur des terres (les Highlands), où ils constituent la richesse la plus valorisée. Ces coquillages ont le statut de biens précieux et fonctionnent comme des monnaies à l'intérieur de certains grands systèmes d'échanges cérémoniels comme le moka dans la région de Mount Hagen (Strathern 1971).

De manière analogue, les Wodani des hautes terres de l'Irian Jaya (partie occidentale de la Nouvelle-Guinée), étudiés par Stéphane Breton, manient une monnaie de coquillage qui n'est rien d'autre qu'une certaine variété de cauris (Breton dir. 2002 : 197-232). Les Wodani se passionnent pour ces coquillages qui ont dû beaucoup voyager pour parvenir jusqu'à eux, au point que la compensation que tout homme doit verser pour obtenir une épouse, passe par un paiement au lignage de celle-ci, fait pour l'essentiel de ces mêmes coquillages qui équivalent chacun à une des parties du corps de l'épouse. Breton n'hésite pas à parler à ce propos de « transsubstantiation» (ibid. : 207).

Chez les Maenge de Nouvelle-Bretagne, étudiés par Michel Panoff, les page (anneaux taillés dans la coquille du tridacne), qui, en fonction des circonstances, constituent des biens précieux ou font office de monnaies, sont également considérés comme provenant d'un lieu lointain et longtemps ignoré; ils proviennent en réalité de Nouvelle-Irlande, mais les réseaux et les modes d'échange qui les ont acheminés chez les Maenge ont brouillé cette origine (Panoff 1980). 


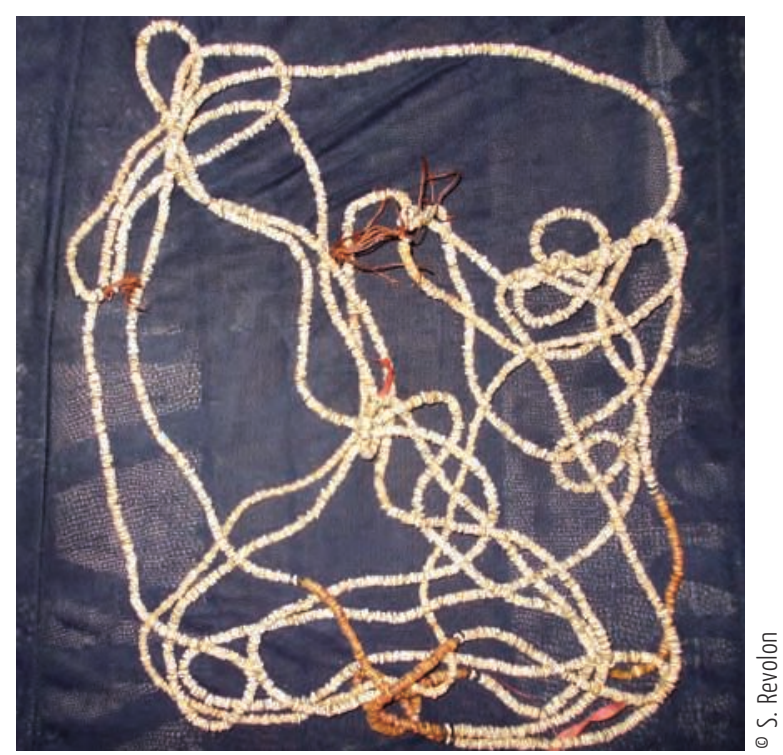

Brasse de perles de coquillage blanches, complétée par des sections de perles rouges. Aorigi, Est des îles Salomon, juillet 2011.
En Nouvelle-Irlande, les Mandak du plateau Lelet, étudiés par Brigitte Derlon, utilisent comme monnaie des enfilades de perles appelées dasilok (Breton dir. 2002 : 157-180). Les mêmes Mandak ont imaginé une origine lointaine à ces biens. Toutefois l'éloignement se fait ici non pas dans l'espace mais dans le temps: les perles ne proviennent pas, au dire des intéressés, du littoral mais des entrailles de Tumbumpo, la plus haute et la plus majestueuse des montagnes qui ceinturent le plateau Lelet.

« Si la distance géographique crée probablement de la valeur en multipliant les transactions et les intermédiaires, observe Derlon, elle a aussi le mérite de faciliter l'ignorance du lieu de production artisanale de l'objet et, partant, la croyance selon laquelle ce dernier est le produit non pas de l'activité humaine mais de quelque source surnaturelle » (ibid. : 158).

Le mythe, ici, tient lieu de voyage.

On le voit, le cheminement et l'échange confèrent de la valeur aux monnaies comme aux objets en général, mais, plus encore, les transfigurent. Le voyage, avec tout ce qu'il suppose de liens complexes entre les objets et les hommes, rend ces objets comme étrangers à eux-mêmes en même temps qu'ils sont étrangers aux acteurs qui les manipulent.

Toutefois, s'il contribue indéniablement à conférer de la valeur aux coquillages qu'affectionnent les hommes, ce caractère d'extranéité - voire d'exotisme - ne suffit pas pour autant à faire de ces coquillages des monnaies: il en fait des biens précieux; ce qui est déjà beaucoup, mais pas suffisant. Nous rappelons ici que l'anthropologie distingue normalement entre les biens précieux et les objets-monnaies, en référence à la notion, proposée dès les années 1950 par Paul Bohannan, de «sphères d'échange »: les premiers s'échangent entre eux au sein d'une même sphère, les seconds s'échangent contre des réalités matérielles ou non fort diverses et franchissent les frontières des sphères d'échange (Dupuy 2008 : 161-164). L'exemple de la kula, étudiée par Bronislaw Malinowski à la fin des années 1910 (Malinowski 1963), et par bien d'autres par la suite, nous montre comment sont échangés deux articles, des colliers de coquillages rouges (appelés soulava) et des brassards de coquillage blanc (appelés mwali). Ces deux articles relèvent de la catégorie des biens précieux (valuables) - catégorie que les habitants de ces archipels situés au large de la pointe sud-est de la Nouvelle-Guinée identifient sous le terne de vaygu'a - et ne sont nullement des monnaies. Néanmoins les procédures créatrices de valeur sont à peu près analogues. Malinowski remarque avec pertinence - mais sans en tirer de conséquence analytique - que l'île de Sanaroa, pourtant située pleinement sur les « routes » de la kula, dans la partie ouest, est exclue des échanges: c'est là précisément que les habitants pêchent et fournissent le coquillage spondyle dont on fait « ces disques rouges qui sont l'un des objets principaux de la richesse indigène » (ibid. : 102). La même chose se passe pour l'île de Misima et celle de Panaeati, au sud-est du « circuit kula », où sont fabriqués les fameux colliers, et qui sont évitées par les grandes routes de l'échange (Leach et Leach dir. 1983 : 453, 475; Damon 1990 [carte du circuit kula]). Autrement dit, ceux chez qui la ressource abonde sont écartés de l'usage de cette même ressource une fois celle-ci transformée en richesse; la proximité ne semble à même de produire que du vulgaire, alors que l'éloignement 
produit de la valeur; car pour ce faire, il faut d'abord, à tout prix, produire de la rareté.

On le constate, le processus de rareté ne suffit pas pour qu'un objet soit reconnu comme monnaie. Il faut en plus qu'intervienne la créance/croyance - rappelons que les deux mots ont la même étymologie - investissant ces objets: c'est là leur dimension « fiduciaire ». Autrement dit, le travail de l'imaginaire collectif force la nature matérielle, pour faire des coquillages autre chose que ce qu'ils sont, et pour qu'ils puissent venir en équivalents à autre chose qu'à eux-mêmes.

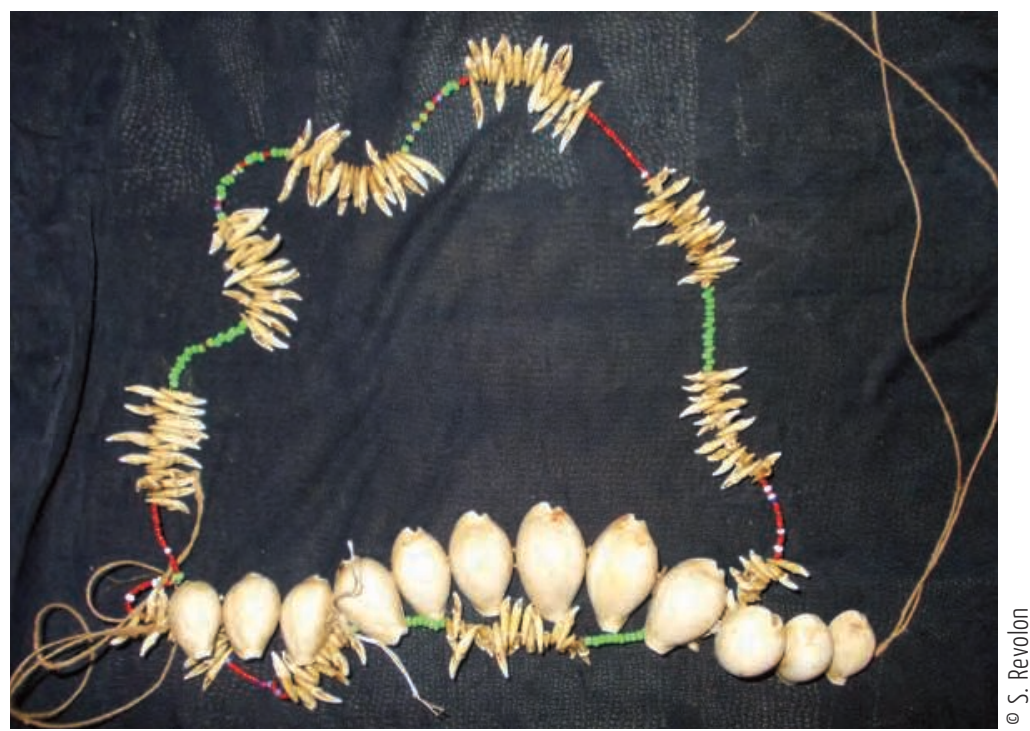

Collier composé de porcelaines, perles de plastique et dents de chauvesouris. Aorigi, Est des îles Salomon, juillet 2011.

\section{Du coquillage à la monnaie}

Dans l'art de convertir un coquillage en monnaie, un exemple célèbre et particulièrement éloquent à ce titre est celui des cauris. L'histoire de ces petits coquillages, qui n'ont l'air de rien mais qui eurent un destin étonnant, est bien connue grâce notamment aux travaux de Jan Hogendorn et Marion Johnson (1986). Ces cauris ont été la principale monnaie de la traite des esclaves: c'est là leur premier et triste titre de gloire, et la clef de leur redoutable succès ${ }^{4}$. Des centaines de millions de petits coquillages, pêchés dans la mer des Maldives (océan Indien) et parvenus sur les côtes d'Afrique après un voyage qui durait une année ou plus, ont fourni une monnaie d'échange quasi inépuisable au trafic négrier. Ils furent d'abord introduits en Afrique noire, réservoir de la main-d'œuvre servile ${ }^{5}$, par les marchands arabes, pionniers dans ce genre de commerce, à partir de l'Afrique du Nord et à travers le Sahara. Plus tard, ils furent embarqués par les compagnies européennes de commerce maritime, qui s'en servaient en tout premier lieu pour lester les cales des navires, jusqu'à Lisbonne, Amsterdam ou Londres. Dans ces métropoles européennes, grands ports d'armement des navires négriers, ils étaient mis en tonneaux et reprenaient la mer dans les soutes des bateaux en direction de l'Afrique de l'Ouest. C'est sur les côtes africaines, et là 

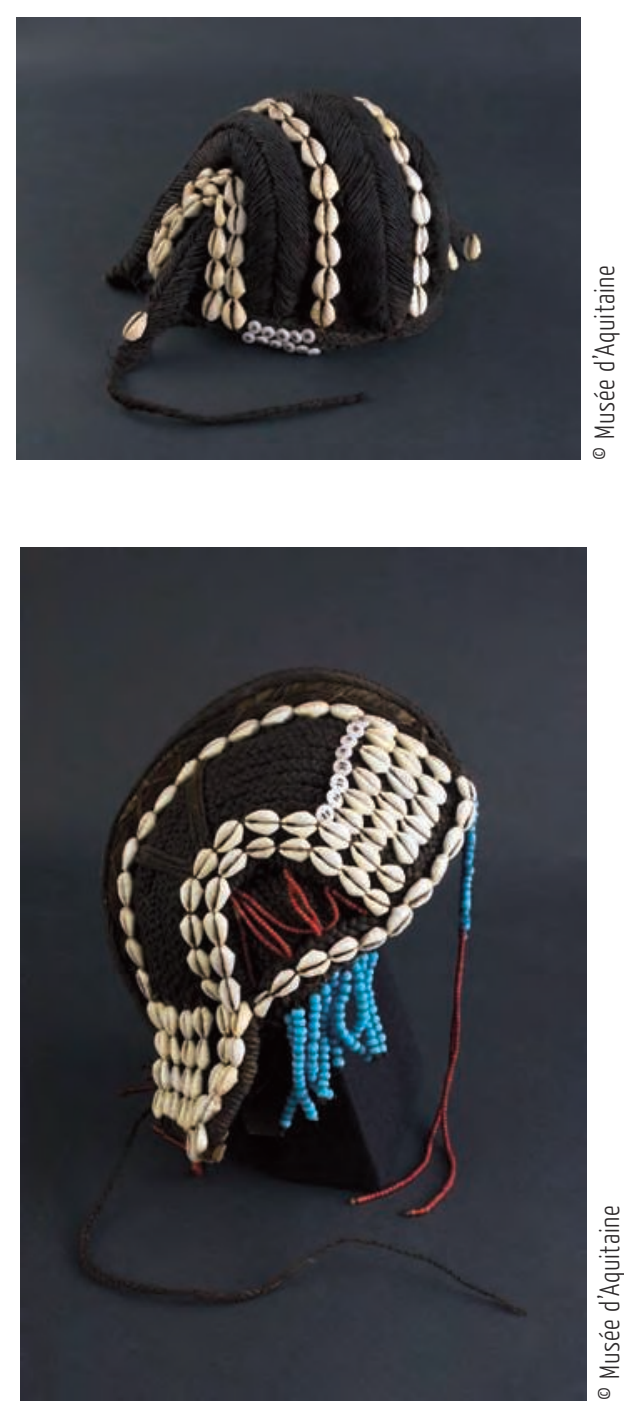

Ci-dessus en haut,

Coiffe Fang (Gabon). Fibres végétales, rotin, cheveux tressés, boutons de nacre et cauris.

Ci-dessus en bas,

Coiffe Fang (Gabon). Fibres végétales, rotin, cheveux tressés, boutons de nacre, perles et cauris. seulement, qu'ils devenaient monnaie. Ils furent utilisés de la sorte avant, pendant et après la traite négrière transatlantique. C'est bien parce qu'ils avaient déjà une valeur reconnue qu'ils ont pu, à partir du Xvi ${ }^{e}$ siècle, lorsque la traite vers l'Amérique a commencé, tenir lieu d'équivalent d'échange fiable et donc fort prisé des trafiquants de toute espèce. Leur existence et leur usage sont attestés pour la première fois par le voyageur-géographe arabe Al-Bakri, à propos de Gao (empire Songhay) en 1067 ; et ils semblent avoir été la monnaie usuelle dans la vaste région du Niger, haut lieu de la ponction des captifs convertis en esclaves, dès le $\mathrm{xI}^{\mathrm{e}}$ siècle. Le commerce par les Européens viendra plus tard, dès le tout début du XvI ${ }^{e}$ siècle avec les Portugais, premiers engagés dans la traite négrière occidentale, d'abord en direction du Portugal puis en direction des Amériques; il se poursuivra au Xvil ${ }^{e}$ siècle avec les Hollandais, les leaders à cette période en matière de commerce maritime, au XviI et surtout au $\mathrm{XVIII}^{e}$ siècle avec les Anglais, puissance commerciale devenue hégémonique. Le XviII ${ }^{e}$ siècle, période d'apogée du trafic des esclaves, suscita une véritable inflation dans l'afflux des cauris (cinquante tonnes par an en moyenne). Robin Law montre quelle était, durant toute cette période, l'importance des cauris dans les transactions commerciales liées à la traite négrière, laquelle était étroitement contrôlée par les autorités étatiques en place (Law 1991). L'auteur précise que les Portugais trouvèrent, à leur arrivée dans cette région, un usage déjà largement répandu de ces coquillages-monnaie (ibid. : 57-58), mais montre également que la traite européenne provoqua une importante inflation: le prix moyen d'un esclave était de 40000 cauris au milieu du XviI ${ }^{e}$ siècle, et du triple un siècle plus tard (ibid. : 176-180).

L'aire d'expansion de ces cauris fut considérable: du Congo au sud (approvisionné depuis Luanda) jusqu'aux « royaumes de la savane " au nord (approvisionnés depuis l'Afrique du Nord), et de l'Ouganda à l'est (approvisionné à partir de la côte swahili) jusqu'au Sénégal à l'ouest (région approvisionnée par les puissances esclavagistes européennes) (Hogendorn \& Johnson 1986 : 101-102).

Le $x_{1}{ }^{e}$ siècle, d'abord avec l'interdiction de la traite négrière puis avec les abolitions successives de l'esclavage dans les colonies d'outre-Atlantique, provoqua un dépérissement du commerce des cauris. Néanmoins, les cauris restaient la monnaie de référence en matière de paiements divers et pour l'achat d'esclaves dans la région du golfe de Guinée. En témoignent, par exemple, les récits de John Duncan, qui voyagea dans le Royaume d'Abomey et les régions voisines, durant les années 1840 , alors même que la traite négrière était déclarée illégale mais qu'elle continuait avec une vigueur inaltérable, notamment à partir du port de Ouidah (Duncan 1847). Entre-temps, ces petits coquillages s'étaient «indigénisés »: ils étaient devenus une monnaie « locale », qui participait du fonctionnement économique d'États fournisseurs d'une main-d'œuvre servile 
(Polanyi 1968 : 280-305), et qui, jusque dans l'intérieur du continent, entrait dans des transactions de toute sorte ainsi que dans le paiement des taxes, des tributs et des compensations matrimoniales. La demande africaine était telle qu'elle occasionna, une fois la traite négrière achevée, une réactivation de l'approvisionnement en provenance des Maldives en passant par Zanzibar. Le trafic de ces cauris ne s'asséchera véritablement que dans les années 1910. Ils continueront toutefois à fonctionner comme monnaie bien au-delà de cette époque. Par exemple, Jack Goody raconte avoir été témoin, au début des années 1980, d’un « changement spectaculaire de la valeur des cauris [...] en tant que monnaie "moderne" » dans la région frontalière entre le Ghana et la Haute-Volta (Goody 2010 : 194).

L'une des clés du phénoménal succès des cauris comme monnaie réside peut-être, du moins pour l'Afrique équatoriale, dans le fait qu'ils sont venus en substitution d'un coquillage local qui remplissait la même fonction. Dans son étude sur l'ancien royaume de Kongo (du xvI ${ }^{e}$ au xviII ${ }^{e}$ siècle), Georges Balandier mentionne l'existence et l'importance de ce coquillage-monnaie: « L'une des premières sources de richesse, pour le roi et les responsables de l'appareil étatique, est l'exploitation exclusive des coquillages-monnaie recueillis aux abords de l'île de Loanda - dénommée par les Portugais, en raison de cette fonction, Itha do Dinheiro ou Itha da Moeda. » Balandier précise: « Les coquillages-monnaie portent, en langue ki-kongo, le nom de nzimbu: nzimbu kitombé et nzimbu a mbudi. Ils sont, selon la classification des naturalistes, de l'espèce Olivancillaria nana Lamarck; il en existe plusieurs variétés, ayant reçu des valeurs différentes, mais leur extension géographique semble limitée aux côtes de l'Angola. Ils doivent être distingués des cauris (Cypraea moneta), fort répandus en Afrique occidentale, qui ne se diffusèrent dans le royaume de Kongo que par l'intervention des traitants; ces nouveaux éléments monétaires, appelés mbési, finirent d'ailleurs par supplanter la monnaie produite localement. ${ }^{6}$ (Balandier 1965 : 121 122). Il apparaît donc qu'il y eut bien ici substitution d'un coquillage par un autre dans une même fonction monétaire. Mais indigènes ou allogènes, les coquillages du royaume de Kongo ressortissaient au bout du compte à un statut identique : l'appareil étatique, s'attribuant l'exclusivité, créait la rareté et transformait des coquillages en objets exceptionnels venus d'ailleurs.

Les cauris semblent la moins « primitive » des «monnaies primitives». De fait, ils étaient pleinement insérés dans une économie marchande internationale, et dans laquelle l'humain lui-même était traité comme une marchandise. Ainsi, ces cauris possédaient les principaux attributs de la monnaie marchande, étatique, comparable aux monnaies

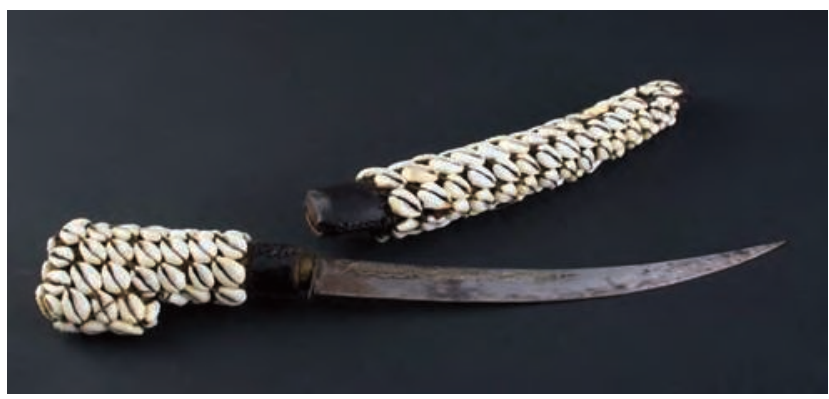

๑ Musée d'Aquitaine
Poignard incrusté de cauris

(Afrique de l'Ouest) 


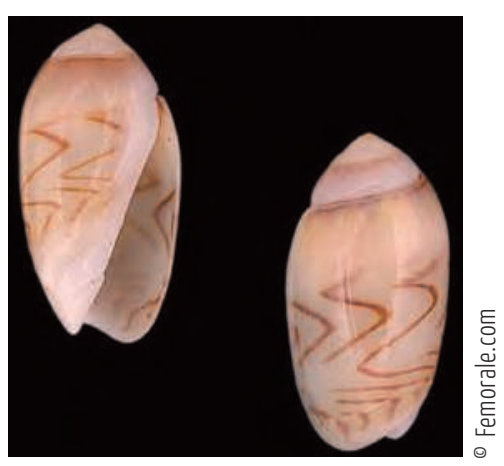

« Olivancillaria nana est aujourd'hui appelée Olivella nana (Lamarck, 1811).

Elle appartient à la famille des Olividae». modernes: l'homogénéité, la durabilité, la portabilité. La divisibilité, dimension importante, faisait défaut, mais cette carence était amplement compensée par une autre vertu: l'impossibilité de contrefaçon; c'est là sans nul doute une autre clef de leur succès. C'est d'ailleurs grâce à toutes ces qualités que l'on put instaurer des systèmes d'équivalence avec les différentes monnaies européennes introduites au cours du XIXe siècle par les économies coloniales (Guyer éd. 1995).

Un autre point doit nous interpeller ici : ces cauris qui voyageaient à fond de cale depuis les Maldives remontaient le long des côtes occidentales de l'Afrique avant de rejoindre les capitales européennes. Ils auraient pu sans doute, dès le trajet aller, servir de monnaie d'échange d'une manière ou d'une autre, mais rien de tel ne se produisait. Il fallait qu'ils transitent d'abord par l'Europe pour revenir ensuite avec leur pleine valeur monétaire. Comme s'il fallait qu'ils fassent un aussi long détour, le temps de cesser d'être des coquillages pour devenir des monnaies. L'Europe, origine mythifiée de toutes les richesses nouvelles et fortement convoitées, était sans doute à même de métamorphoser de vulgaires coquillages en monnaies capables d'acheter jusqu'à des êtres humains.

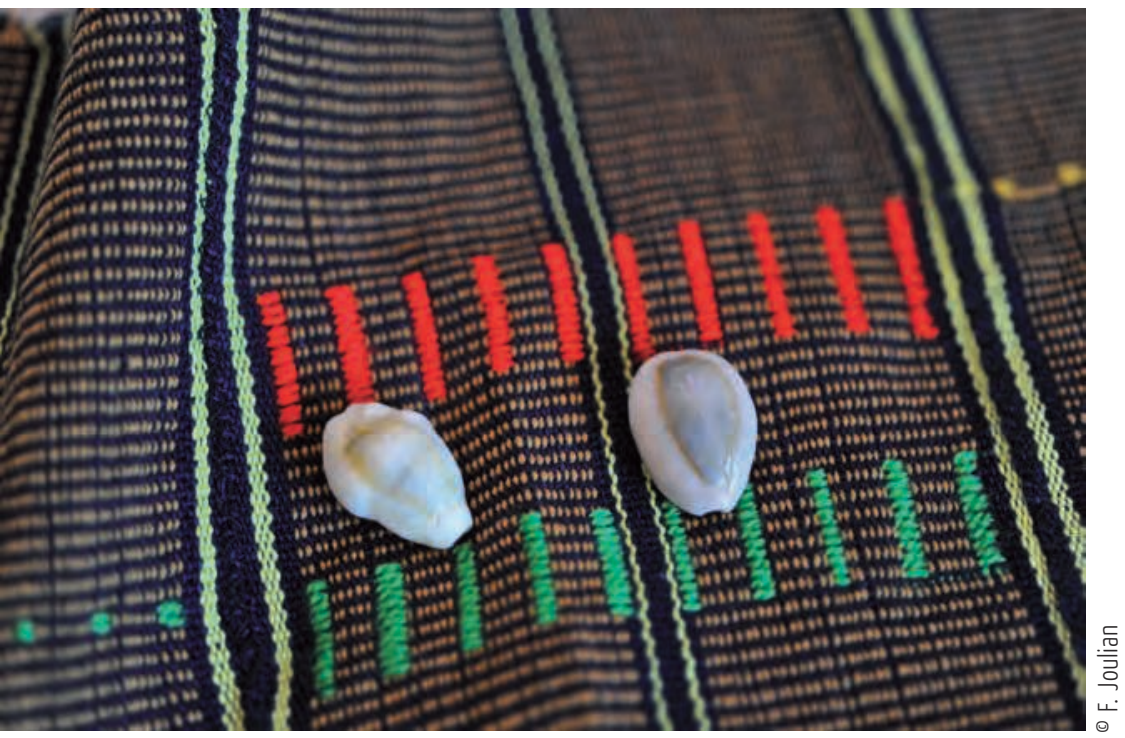

\section{De la monnaie au coquillage}

L'étonnant périple du petit coquillage, parti des Maldives, passant par les grands ports de l'Europe occidentale et parvenant - en de sinistres circonstances - jusqu'en Afrique, où il s'implante durablement, ne s'arrête pas là...

Sur les pourtours de l'Amazonie, en Guyane française et au Surinam, se trouvent six communautés de Noirs Marrons, autrement appelés Bushinenge - terme autrefois infamant, correspondant à l'appellation « Nègres des bois », usitée jadis en français, mais désignation revendiquée aujourd'hui par les intéressés, après un retournement 
de charge symbolique, pour une affirmation identitaire. Ceux qui ont constitué ces communautés au Xvin ${ }^{e}$ siècle étaient issus de la traite négrière, de l'esclavage sur les plantations (de l'ancienne Guyane hollandaise, devenue le Surinam) et du marronnage. Afin d'échapper à leur condition servile, ils avaient fui vers l'intérieur des terres dans l'intention de recouvrer la liberté et de trouver refuge dans la forêt. Assez nombreux pour composer des ensembles organisés, ils ont façonné des sociétés à partir d'une matrice sociale et culturelle en provenance directe d'Afrique de l'Ouest, à partir d'une Afrique qu'il leur fallut cependant retraiter voire réinventer, en raison de la diversité culturelle des sociétés de provenance, et du brassage que le système colonial s'était employé à opérer. Cette Afrique originelle a été en quelque sorte synthétisée. En matière linguistique, l'on dut forger sur place une langue, à partir d'une base lexicale empruntant pour l'essentiel à l'anglais. Ces communautés marronnes forment des sortes d'enclaves africaines en terre d'Amérique, qui contrastent fortement avec les sociétés amérindiennes qui se situent dans les mêmes parages.

Il se trouve que parmi ces Noirs Marrons des objets ont une valeur et une portée toutes particulières: ce sont les cauris. La présence de ces coquillages en Amérique est plutôt inattendue, mais on constate qu'ils ont fait, eux aussi, le voyage transatlantique. Ils ont accompagné les hommes qui, en Afrique, les connaissaient bien et les utilisaient. Et ils sont toujours utilisés de nos jours dans les Guyanes par les descendants de ceux dont les ancêtres furent probablement vendus contre quelques poignées de ces mêmes cauris.

On se procure ces coquillages le plus souvent dans des boutiques spécialisées de Paramaribo; on rencontre en effet dans cette capitale des magasins proposant, sous les formes les plus diverses, des articles entrant dans la composition des obia, c'est-àdire des forces supérieures que l'on tente d'actionner afin d'intervenir, en bien ou en mal, sur les humains, les événements, et plus largement sur le monde extérieur (visible et invisible). Chez les Boni (ou Aluku) qui habitent le cours moyen du Maroni, par exemple, ces cauris vont permettre de fabriquer des «bijoux » (colliers, ceintures), qui servent non pas tant de parures que de protections. Des individus, hommes ou femmes, gardent ou portent sur eux un cauri dans la fente duquel est fixée une plume d'ara (que l'on peut se procurer dans les mêmes boutiques de Paramaribo). Cet objet, en unissant un coquillage emblème de l'Afrique et une plume de l'oiseau emblématique de l'Amérique du Sud, présente à lui tout seul un résumé du destin des Noirs Marrons.

La façon la plus traditionnelle d'utiliser les cauris consiste à confectionner un collier ou une ceinture avec de fines lanières de tissu que l'on tresse en traversant un cauri que l'on a préalablement percé, cauri dans lequel on coince une plume d'ara dont on coupe la « tête ». On porte ce collier ou cette ceinture comme protection, particulièrement quand on s'expose à un danger - par exemple, un voyage lointain hors de sa communauté est une circonstance considérée comme dangereuse. On porte également ce collier ou cette ceinture lorsque l'on est malade. La maladie est représentée et vécue par la personne comme l'effet d'un éloignement de son akaa (i. e. l'âme ou, plus

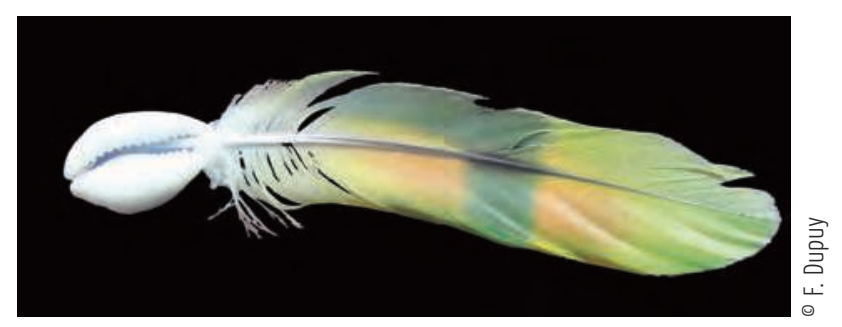




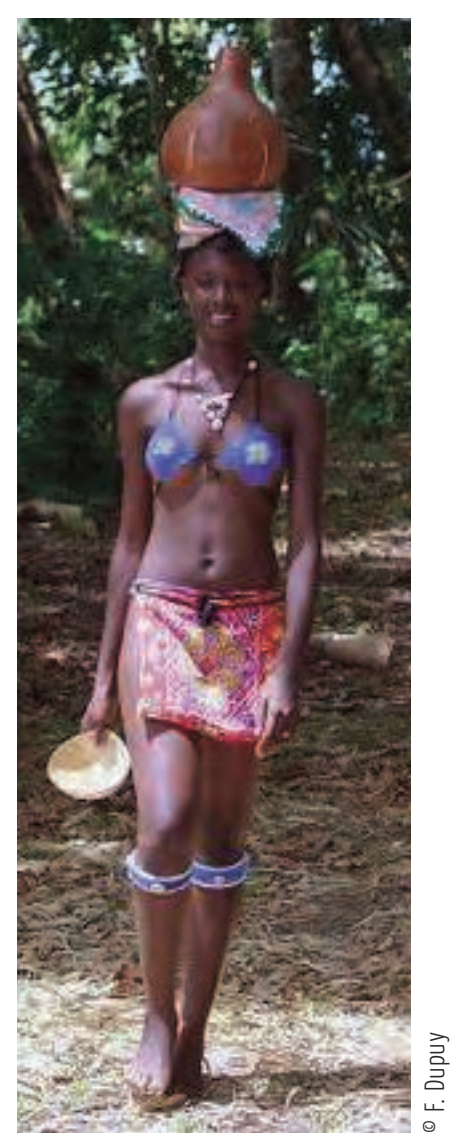

Jeune fille Boni (Guyane) vêtue en costume « néo-traditionnel ». Des cauris figurent dans sa parure. précisément, le principe vital, en lien avec les ancêtres). Collier ou ceinture permettent alors de rappeler son akaa auprès de soi, pour éviter la mort. Un autre cas de figure est la défense contre la sorcellerie (wishi), qui joue un rôle omniprésent dans la vie sociale, et dont on doit protéger tout à la fois les vivants et les ancêtres - raison pour laquelle, à la mort d'un individu, on interroge son cadavre, un peu comme on le fait en Afrique occidentale. Deux types de collier/ceinture existent: l'un est dit sama mofu tetei (il protège de la sorcellerie envoyée par quelqu'un qui veut nuire), l'autre akaa tetei (il intervient pour rappeler le akaa). On voit donc que les Noirs Marrons utilisent le cauri comme protection, face à ce qu'ils considèrent comme les deux menaces majeures: la sorcellerie et la maladie. La confection de ces colliers et de ces ceintures et la manipulation de ces cauris sont nécessairement l'affaire de personnes compétentes en matière d'obia - des "maîtres des obia » (obia bashi), connaissant bien le «langage des obia» (obia tongo).

Dans le transfert transatlantique, le mot a suivi la chose: le terme vernaculaire que les Noirs Marrons des Guyanes utilisent pour désigner ces cauris ainsi recyclés est celui de papa moni. Cette expression signifie littéralement « monsieur monnaie »: le mot papa indique une marque de respect que l'on adresse à tous les hommes d'un certain âge, de sa propre parenté ou non, et dont l'équivalent français est « monsieur ». Quant au terme moni, il vient bien évidemment du terme correspondant en anglais, auquel les langues marronnes de ces régions ont puisé, nous l'avons dit, l'essentiel de leur stock lexical. Par cette désignation, les Marrons des Guyanes réfèrent explicitement au passé monétaire des cauris, mais ils en ont détourné le sens. Si certains parmi eux connaissent ce passé monétaire, ils n'en cultivent aucunement la mémoire; et jamais en Amérique ils n'ont utilisé ces petits coquillages pour payer quoi que ce soit. Symbole du malheur pour les esclaves vendus et achetés sur les côtes africaines, le cauri est devenu support de protection pour leurs descendants en terre américaine. Cette ancienne monnaie démonétisée et américanisée est ainsi devenue un recours « magico-religieux » pour affronter la vie.

Une culture est en mesure de défaire ce qu'elle a fait. Les Amériques noires ont recyclé ce que l'Afrique noire avait intégré et façonné. Et les Africains transplantés en Amérique ont « déconstruit » une monnaie africaine, qui au demeurant l'était si peu...

\section{$\&$}

En forme de conclusion, nous insisterons sur deux ensembles de réflexions. En premier lieu, nous observerons que les coquillages nous montrent toute la force des conventions sociales. Au départ, quoi de plus banal qu'un coquillage? Pourtant bien des sociétés ont décrété qu'un coquillage pouvait être la forme suprême de la richesse, tels les indigènes de la région de Mount Hagen, dans les Western Highlands de Papouasie-Nouvelle-Guinée, avec leurs pearlshells ou les Massim, dans les îles du sud-est de la Nouvelle-Guinée, avec leurs vaygu'a. Pour convertir un coquillage en bien précieux et/ou en monnaie, il faut le soumettre à un énorme travail idéologique, par lequel, au bout du compte, l'imaginaire l'emportera sur le réel. La valeur résultera de cette capacité à transformer les coquillages en autre chose que ce qu'ils sont. 
L'éloignement, réel ou fictif, de leur origine et le voyage plus ou moins long auquel on les a soumis avant d'être, en leur lieu de destination, dans leur pleine efficacité sociale et symbolique, contribuent de toute évidence à cet incrément de valeur.

En second lieu, comme nous avons tenté de le montrer ailleurs (Dupuy 2008, 2009), les monnaies offrent un sujet privilégié pour comprendre les rapports sociaux; elles expriment, condensent et cristallisent les rapports sociaux; elles en sont comme un précipité. C'est bien la raison pour laquelle une monnaie, de coquillage ou de toute autre chose, ne dit rien en elle-même mais dit beaucoup par elle-même en fonction des rapports sociaux dans lesquels on l'implique. Si les monnaies traditionnelles perdurent en bien des lieux alors même que la monnaie marchande a déferlé depuis plus ou moins longtemps, c'est parce qu'elles réalisent des relations non marchandes, comme le paiement de la compensation matrimoniale ou de la compensation mortuaire. Elles payent pour des personnes vivantes ou mortes dans des sociétés où, en revanche, on ne peut rémunérer la force de travail de ces mêmes personnes. À l'opposé, les cauris dans l'Afrique subsaharienne, partie prenante d'une économie marchande déjà mondialisée, ont permis durant des siècles d'acheter non seulement la force de travail mais les êtres humains eux-mêmes comme s'il s'agissait de biens meubles. En d'autres termes, le cauri chez les Wodani de l'Irian Jaya n'a rien à voir avec celui des trafiquants négriers. C'est bien le système économique et social dans lequel elle est agie et le type de relation qu'elle réalise qui fondent la nature réelle d'une monnaie et son efficacité sociale. La monnaie n'est rien d'autre que ce qu'on lui fait faire.

Tout comme les gros coquillages ne renvoient aux enfants auxquels on les offre qu'un bien illusoire bruit de la mer, les coquillages ayant intégré la société des hommes ne nous parlent finalement que fort peu de leur élément liquide originel. Ils nous renvoient en revanche un écho assez fidèle de ce que nous sommes. 


\section{NOTES}

Photo d'ouverture: Une jeune fille ajoute une brasse de perles et de dents de chauves-souris à d'autres brasses de billets de banque et de perles de coquillage lors du paiement d'un prix de la fiancée. Aorigi, Est des îles Salomon, juillet 2011. (Cliché S. Revolon)

1. L'Amérique en revanche ignore presque totalement les monnaies en général et les coquillages en particulier. Le vaste ensemble amazonien, notamment, se présente comme un « vide monétaire », à propos duquel nous avons essayé ailleurs de fournir quelques clés d'explication (Dupuy 2012). Toutefois, il convient de signaler pour l'Amérique du Nord le célèbre wampum des Iroquois, une monnaie fabriquée à partir de coquillages provenant de la Côte Est (Graeber 2001 : 117-149).

2. Sur ces questions fort complexes mais quelque peu en dehors du champ de cette contribution, nous renvoyons à d'autres écrits (Dupuy 2008, 2009, 2012).

3. Karl Polanyi considère qu'une monnaie est un « système sémantique » (Polanyi 1968: 281).
4. Cette section propose une version modifiée et amplifiée d'un texte antérieur (Dupuy 2009 : 133-135).

5. Rappelons que les témoignages les plus anciens concernant la traite transsaharienne remontent au $\mathrm{VII}^{\mathrm{e}}$ siècle, et qu'elle semble affecter l'Ouest africain dès le Ixe siècle (Meillassoux 1986: 44).

6. Depuis les écrits de Balandier, les classifications et les désignations scientifiques ont changé. Le cauri est devenu Monetaria moneta, et l'autre coquillage, de la famille des Olividae, est devenu Olivella nana; la distribution de ce dernier est limitée aux côtes ouest-africaines, entre le Gabon et le sud de l'Angola. Je dois ces précisions taxonomiques à Philippe Bouchet, du Muséum National d'Histoire Naturelle (communication personnelle), que je remercie.

\section{REMERCIEMENTS}

Nous tenons tout particulièrement à remercier Sandra Revolon (Aix-Marseille Université / Credo) de son aide précieuse pour l'illustration de cet article.

\section{RÉFÉRENCES}

Balandier, G. 1965 La Vie quotidienne au royaume de Kongo du XVI au XviI e siècle. Paris: Hachette.

Breton, S. (dir.) 2002 Questions de monnaie. L'Homme 162.

Connell, J. 1977 The Bougainville Connection: Changes in the Economic Context of Shell Money Production in Malaita. Oceania XLVIII(2): 81-101.

Cooper, M. 1971 Economic Context of Shell Money Production in Malaita. Oceania XLI(4): 266-276.

Damon, F. 1990 From Muyuw to the Trobriands. Transformations Along the Northern Side of the Kula Ring. Tucson: The University of Arizona Press.

Duncan, J. 1847 Travels in Western Africa in 1845 and 1846, Comprising a Journey from Whydah through the Kingdom of Dahomey, to Adofoodia, in the Interior. Londres: Hard Bentley, 2 vol.

Dupuy, F. 2012 Monnaies « primitives » : polysémie et plurifonctionnalité. In Pion, P. et Formoso, B. (éds), Monnaie antique, monnaie moderne, monnaies d'ailleurs... Métissages et hybridations, Colloques de la Maison René-Ginouvès, 8. Paris : De Boccard : 45-53.

— 2009, Les « monnaies primitives ». Nouvelles considérations. L'Homme 190: 129-151.

- 2008 [2001] Anthropologie économique. Paris: Armand Colin.

Einzig, P. 1949 Primitive Money in its Ethnological, Historical and Economic Aspects. Londres: Eyre et Spottiswoode.

Goody, J. 2010 [2006] Le Vol de l'histoire. Comment l'Europe a imposé le récit de son passé au reste du monde. Paris: Gallimard.

Graeber, D. 2001 Toward an Anthropological Theory of Value. The False Coin of Our Own Dreams. New York: Palgrave. 
Guo, P. 2007 "Making Money": Objects, Productions, and Performances of Shell Money Manufacture in Langalanga, Solomon Islands. In Michael H. H. Hsiao (dir.) New Frontiers of Southeast Asia and Pacific Studies. Taipei: Center for Asia-Pacific Area Studies, Academia Sinica: 211-240.

Guyer, J., I. (dir.) 1995 Money Matters. Instability, Values and Social Payments in the Modern History of West African Communities. Portsmouth/Londres: Heinemann/Currey.

Hogendorn, J., Johnson, M. 1986 The Shell Money of the Slave Trade. Cambridge: Cambridge University Press.

Law, R. 1991 The Slave Coast of West Africa 1550-1750. The Impact of the Atlantic Slave Trade on an African Society. Oxford: Clarendon Press.

Leach, J., Leach, E. (dir.) 1983 The Kula. New Perspectives on Massim Exchange. Cambridge: Cambridge University Press.

Malinowski, B. 1963 [1922] Les Argonautes du Pacifique occidental. Paris: Gallimard.

Meillassoux, C. 1986 Anthropologie de l'esclavage. Le ventre de fer et d'argent. Paris: PUF.

Panoff, M. 1980 Objets précieux et moyens de paiement chez les Maenge de Nouvelle-Bretagne. In L'Homme $\mathrm{XX}(2): 5-37$.

Polanyi, K. 1968 Primitive, Archaic, and Modern Economies, essais édités par George Dalton. New York: Anchor Books.

Quiggin, A., H. 1949 A Survey of Primitive Money. The Beginning of Currency. Londres: Methuen.

Strathern, A. 1971 The Rope of Moka. Big-men and Ceremonial Exchange in Mount Hagen, New Guinea. Cambridge: Cambridge University Press.

\section{RÉSUMÉ}

Des coquillages de valeur. Dans un premier temps, le texte présente quelques exemples océaniens montrant que le voyage et l'éloignement du lieu d'origine font subir aux coquillages une métamorphose produisant une valeur symbolique jusqu'à faire de ces derniers des monnaies. Ensuite, nous présentons le cas hors norme des cauris qui, en provenance des Maldives et passés par les pays européens, connurent un succès spectaculaire en Afrique à travers la traite négrière. Enfin, nous suivons ces mêmes cauris pour un dernier voyage en Amérique, où, dans la région des Guyanes, ils ont perdu leur valeur monétaire mais sont devenus auprès des Noirs Marrons un support de protection magique.

\section{ABSTRACT}

Shells of value. At first, the paper presents some oceanian examples showing that travel and the remoteness of the place of origin are bringing to the shells a metamorphosis producing up a symbolic value able to make them money. Then, we present the extraordinary case of cowries, which from the Maldives and passed through European countries experienced a spectacular success in Africa through the slave trade. Finally, we follow these cowries for a more trip to America, where, in the region of the Guianas, there have lost their monetary value but have become among the Maroons support of magical protection.

\section{MOTS CLÉS}

Monnaies «primitives », valeur, paiement, échange, Océanie, Afrique, Amériques noires

\section{KEYWORDS}

Primitive money, value, payment, exchange, Oceania, Africa, Black Americas 